\section{Theoretical reality}

\author{
S. Roy Caplan
}

Water Movement Through Lipid Bilayers, Pores, and Plasma Membranes: Theory and Reality. Vol. 4, Distinguished Lecture Series of the Society of General Physiologists. By Alan Finkelstein. Wiley: 1987. Pp. 228. \$39.95, £36.65.

AN eminent reviewer of the time recommended that Guggenheim's treatise Thermodynamics should be subtitled Pride and Prejudice. Without implying a parallel, this remark all too frequently comes to mind while reading Dr Finkelstein's monograph. The pity is that what might have been an excellent source of information is marred by the author's crotchety preconceptions in assessing the contributions of nonequilibrium thermodynamics to his field.

The work is divided into three parts. Part I ("Theory") accounts for virtually half of the text, encompassing both porous and non-porous ("oil") membranes. The remaining half is apportioned evenly between Parts II and III. Part II is devoted to a detailed treatment of the pores generated in lipid bilayer membranes by antibiotics. while Part III deals with the red cell membrane and with epithelia. The latter half of the book contains the most critical assessment of data on physiological water movement available. It is, however, coloured by the views expressed in Part I.

As Dr Finkelstein points out, there is currently no satisfactory theory to describe solvent flow in pores of molecular dimensions. He avoids mentioning that the concept of pressure in such pores is illdefined, but rather presents us with explanatory diagrams displaying pressure profiles. These intuitive schemes may certainly provide a mental crutch. For the author, however, they constitute reality, prompting him to castigate nonequilibrium thermodynamics for its ability "to obfuscate the physics underlying osmotic transport through a porous membrane". Thus osmotic flow across a membrane separating solvent and a solution of an impermeant solute, both at the same pressure, is said to be driven simply by a pressure gradient within the pore. If this is the whole story, surely the opposing infinite pressure gradient (as drawn) at the pore exit also needs explicit consideration.

The picture is extended to the wellknown experiment by Meschia and Setnikar supposedly demonstrating water flow in the wrong direction across a collodion membrane separating moderately concentrated urea and dilute dextran at the same pressure. Osmosis proceeds from urea to dextran, against the chemical potential gradient for water. The notion that solute flux may be coupled to, and hence may drag, solvent flux "completely distorts reality" in Dr Finkelstein's eyes. Not so, he says, water drags urea because the pressure gradient within the pore forces the solution towards the dextran side. How it forces it out of the pore against the large opposing pressure gradient is left for the hapless reader to figure out.

In case of any lingering doubts, Dr Finkelstein asks us to consider a huge sewer pipe with the urea solution at one end, under slight pressure, and pure water at the other. This is claimed to be "exactly comparable" to what occurred in the Meschia and Setnikar experiment. But the comparison is preposterous. The sewer pipe is not of molecular dimensions, and

\section{Fighting fit}

John R. Krebs

Animal Conflict. By Felicity Huntingford and Angela Turner. Chapman \& Hall: 1987. Pp.448. Hbk £30, \$59.95; pbk $£ 14.95, \$ 27.50$.

THIS useful introductory book covers much the same ground as Roger N. Johnson's admirable Aggression in Man and Animals (W.B. Saunders, 1972), although it is of course much more up to date. It gives a balanced review of aggression and related behaviours in the animal kingdom and considers various theories about both the causes of aggression and its functional significance. The final chapter moves with due caution from animal studies to human aggressive behaviour. Probably the main messages from the book are: (a) in wild animals aggressive behaviour is not a pathology but part of normal adaptive behaviour; (b) different kinds of aggression (predatory, defensive, competitive) may serve different functions and have different causes; and (c) simple general theories on causes of aggression, popular in the past, are likely to be wrong.

In the $1960 \mathrm{~s}$, perhaps not unrelated to the peace movement and rise of flower power, there was a fashion for global theories of human aggression: it was argued that aggression is caused by frustration (never thwart your kids and they will grow up as peace-loving citizens), by pain (do away with corporal punishment and kids will stop being aggressive), by copying role models (cut out violent television movies) and so on. We now know that all these ideas are too simple as overall theories of aggression. A widely publicized ethological contribution to $1960 \mathrm{~s}$ theorizing was the one so forcefully and eloquently expressed by Konrad Lorenz in his classic book Das sogennante Böse (Borotha-Schoeler, 1963), tamely translated into English as On Aggression (Methuen, 1966). According to the no opposing pressure gradient exists anywhere. If Dr Finkelstein had read the papers of Kedem and Katchalsky he quotes more closely, he might have noticed that the force driving volume (not solvent) flow in their formulation is the overall pressure difference less the osmotic pressure difference of impermeant solute. This correctly describes the volume flow in both the collodion membrane and the sewer pipe. Is this obfuscation?

Nevertheless, I would urge all interested in this problem to buy Dr Finkelstein's book. Nothing better is around.

S. Roy Caplan is a Professor in the Department of Membrane Research, The Weizmann Institute of Science, 76100 Rehovot, Israel.
Lorenzian view, aggression in animals and man is the expression of an inbuilt drive that wells up inside and has to find release in one form or another. The longer it is since you had a meal, the hungrier you feel, and, according to Lorenz, aggressive feelings build up in the same way since your last fight. Lorenz's view, based in part on the correct notion that aggression, like feeding, is normally an adaptive and essential component of an animal's daily life, is still very influential. Only a couple of months ago I read a newspaper article on football crowd violence which argued that to suppress violence on the terraces would be merely to cause it to erupt elsewhere - the classic Lorenzian theory.

Sidestepping the difficult issue of how and if at all football hooligan aggression is related causally or functionally to other kinds of fighting, one thing is clear: ethological research has shown during the past 20 years that the Lorenzian drive model of aggression in animals is wrong. As Huntingford and Turner make clear, aggression in animals is a form of competition (usually with members of the same species) for limited resources of food, mates or shelter. The evidence shows that the extent to which animals compete by fighting for resources, as opposed to competing by non-aggressive means such as simple exploitation, depends on ecological circumstances. Both within and between species, the extent of aggressive competition depends on the distribution of resources: when they are localized, animals tend to defend them and fight; when resources are scattered or occur unpredictably in time and space, animals tend not to compete by aggression. If anyone wants to use ethological arguments as a starting point for building a new general theory of aggression, this view of aggression as a flexible strategy, rather than the notion of an inevitable internal build-up, should be the starting point.

John R. Krebs is a Lecturer at the Edward Grey Institute of Field Ornithology, Department of Zoology, University of Oxford, South Parks Road, Oxford OXI $3 P S, U K$ 